\title{
Building sustainable and consequential research capacity within a global alliance of paediatric surgical centres
}

\author{
Suraj M. Gandhi ${ }^{1}$ (I) Krithi Ravi $^{2} \cdot$ Fatumata Jalloh-PA-R $^{3} \cdot$ Noel Peter $^{4} \cdot$ Kokila Lakhoo $^{4}$
}

Accepted: 6 January 2021 / Published online: 25 January 2021

(c) The Author(s), under exclusive licence to Springer-Verlag GmbH, DE part of Springer Nature 2021

Keywords Paediatric surgery $\cdot$ Global surgery $\cdot$ Research capacity

The Global Health Research Group on Children's NonCommunicable Diseases recently launched the PaedsCancer (COVID) study to measure the impact of the COVID-19 pandemic on paediatric cancer management worldwide. At the time of writing, collaborators hail from over 85 countries, of which over $64 \%$ are low- and middle-income countries (LMICs). Global Children's NCDs recognises the risk of propagating HIC dependence in global collaborative paediatric surgery research; LMICs, where the surgical need is greatest, accounted for just $15 \%$ of surgical research reports from the 35 highest output countries from 2009 to 2013 [1, 2].

Locally meaningful research is driven by stakeholders with the deepest comprehension of the community's needs [1-3]. To promote LMIC research capacity, we will offer our collaborators-from students and allied health professionals to senior clinicians-training in translational research skills.

We endeavour to:

Suraj M. Gandhi, Krithi Ravi and Fatumata Jalloh-PA-R are the joint first authors.

Suraj M. Gandhi

sg460@student.le.ac.uk

1 George Davies Centre, Leicester Medical School, Lancaster Rd, Leicester LE1 7HA, UK

2 Heartbeat Education Centre, Southampton General Hospital, University Hospital Southampton NHS Foundation Trust, Tremona Road, Southampton SO16 6YD, UK

3 Gloucestershire Hospitals NHS Foundation Trust, Great Western Rd, Gloucester GL1 3NN, UK

4 Nuffield Department of Surgical Sciences, John Radcliffe Hospital, Oxford University Global Surgery Group, Headington, Oxford OX3 9DU, UK
- Empower participants with research and audit skills to develop their own projects and implement their findings to affect local practice.

- Mentor participants through the development, implementation, write-up, and dissemination of their project.

- Enhance their career development by providing a formal qualification.

We aim to achieve the above through a web-based research training course, taught using examples throughout to illustrate research principles. Recorded webinars will be uploaded online to facilitate access for those with time constraints and Internet bandwidth limitations. Interactive sessions will be rotated across time zones for equity of access.

Webinar translations will be produced for non-English speakers, to encourage locally relevant research output resulting from "consequential and often non-academic conversations" in non-English languages [3]. Web-based interactive small group sessions will allow participants to "learn by doing" and promote the formation of academic relationships and collaborations [4].

The committee structure, with a senior advisory team experienced in conducting research in LMIC settings and a programme delivery committee of motivated early career global surgery researchers, reflects our aim of facilitating the transfer of research skills and experience from LMIC-based clinician-academics to collaborators [4].

Local collaborators are best equipped to tailor research agendas to local needs, commit to sustainable research programmes, and affect policy through contact with relevant stakeholders [2]. Emphasising capacity building helps avoid the "one size fits all" approach to surgical research [1]. Our research capacity building course aspires to empower participants with the tools and mentorship necessary to design and implement original research "from a local pose for a 
local gaze" [3], resulting in safer, more accessible and higher quality surgical services in LMICs.

The authors have no conflicts of interest to declare that are relevant to the content of this article. No funding was received to assist with the preparation of this manuscript.

Author contributions SG, KR and FJ drafted the letter. NP and KL made edits to the final letter. KR, FJ, NP and KL devised the research capacity building programme.

\section{References}

1. Cunningham A, Stephens C, Ameh E, Mshelbwala P, Nwomeh B, Krishnaswami S (2019) Ethics in global pediatric surgery: existing dilemmas and emerging challenges. World J Surg 43(6):1466-1473

2. Meara J, Leather A, Hagander L et al (2015) Global Surgery 2030: evidence and solutions for achieving health, welfare, and economic development. Lancet 386(9993):569-624

3. Abimbola $S$ (2019) The foreign gaze: authorship in academic global health. BMJ Global Health 4(5):e002068

4. Färnman R, Diwan V, Zwarenstein M, Atkins S (2016) Successes and challenges of North-South partnerships - key lessons from the African/Asian regional capacity development projects. Global Health Action 9(1):30522

Publisher's Note Springer Nature remains neutral with regard to jurisdictional claims in published maps and institutional affiliations. 